\title{
CLINICAL PATHWAY FOR THE PREVENTION OF PERIOPERATIVE HYPOTHERMIA IN THE CANDIDATE FOR MAJOR ONCOLOGICAL SURGERY: EFFECTIVENESS AND AREAS OF IMPROVEMENT.
}

C. Ferraris, V. Karakotch, E. Malja, M. Boezi, S. Siligardi, G. Cipriani.

Complex Operative Unit of Anesthesia and Reanimation - San Pietro Hospital - Rome

Aim. Unintended periopertive hypothermia is common in surgical patients and is related to significant morbidity and mortality. Monitoring of body temperature and avoiding the unintended perioperative hypothermia using active warming techniques are key factors of prevention of complications including surgical infection, delayed wound healing, adverse myocardial events, and increased bleeding. This study intends to verify the effectiveness of an internal management program, dedicated to major oncological surgery, aimed at the prevention of perioperative hypothermia and planned according to the indications contained in the guidelines and the best practices that deal with the topic.

Materials and methods. The care path has been applied to 200 patients. In all the perioperative phases, the central temperature of the patients was monitored with not invasive zero-heat-flux technology Bair Hugger system. Patients received an optional preoperative and mandatory intraoperative warm up a forced air system Bair Hugger in association with underbody blankets. Infusion fluids were actively heated in all the interventions using the standard Ranger IV system. For each patient the demographic and anamnestic parameters were recorded as well as quantitative and qualitative indicators indispensable for a correct evaluation of the patients outcome.
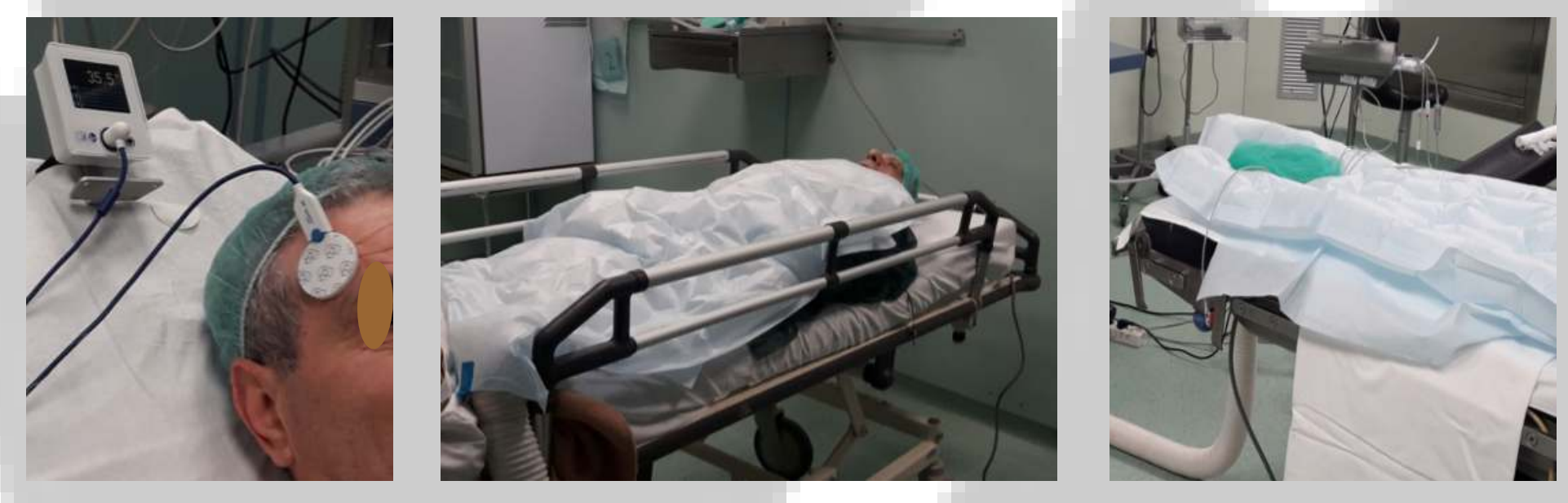
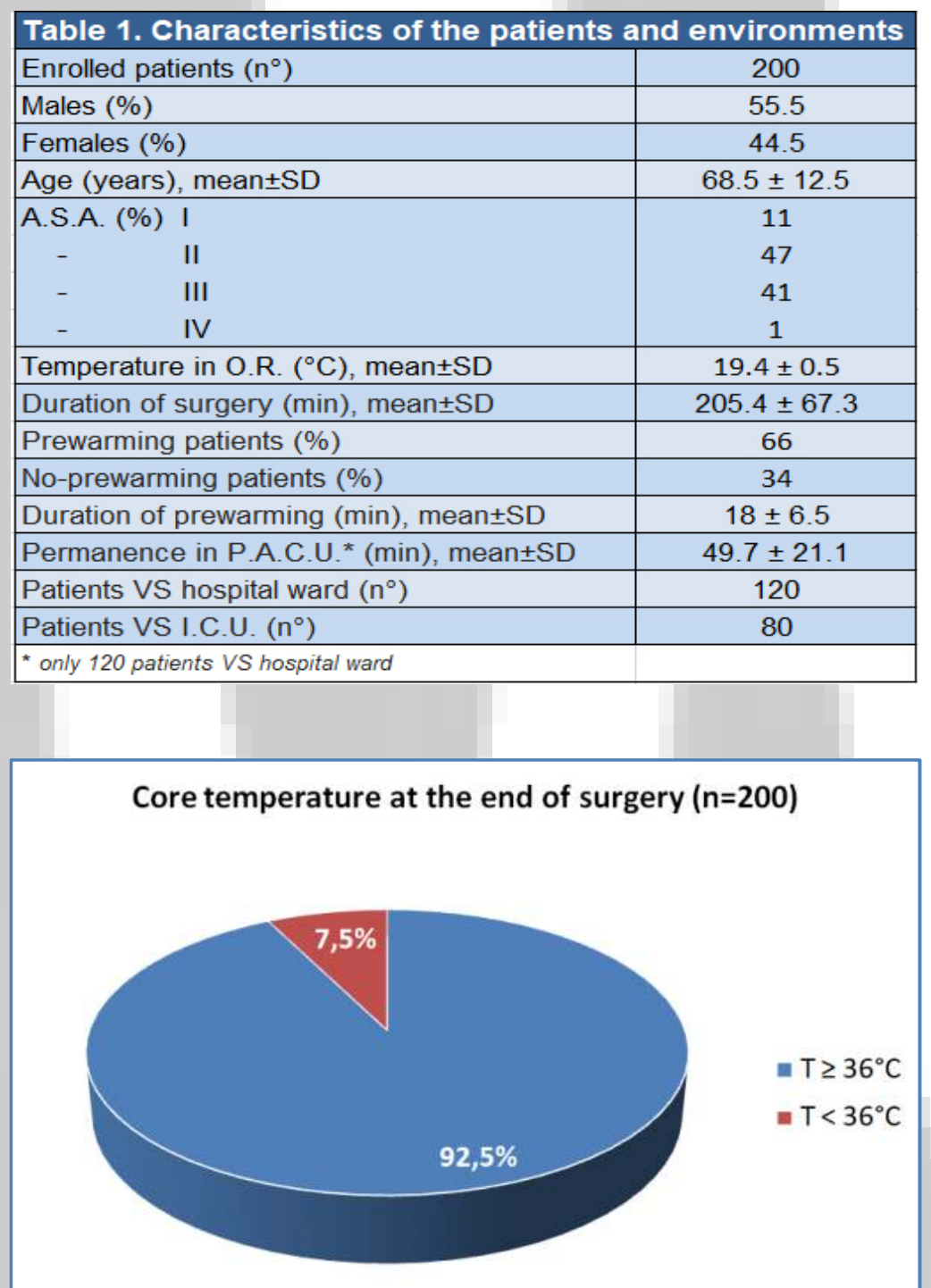

Average core body temperature

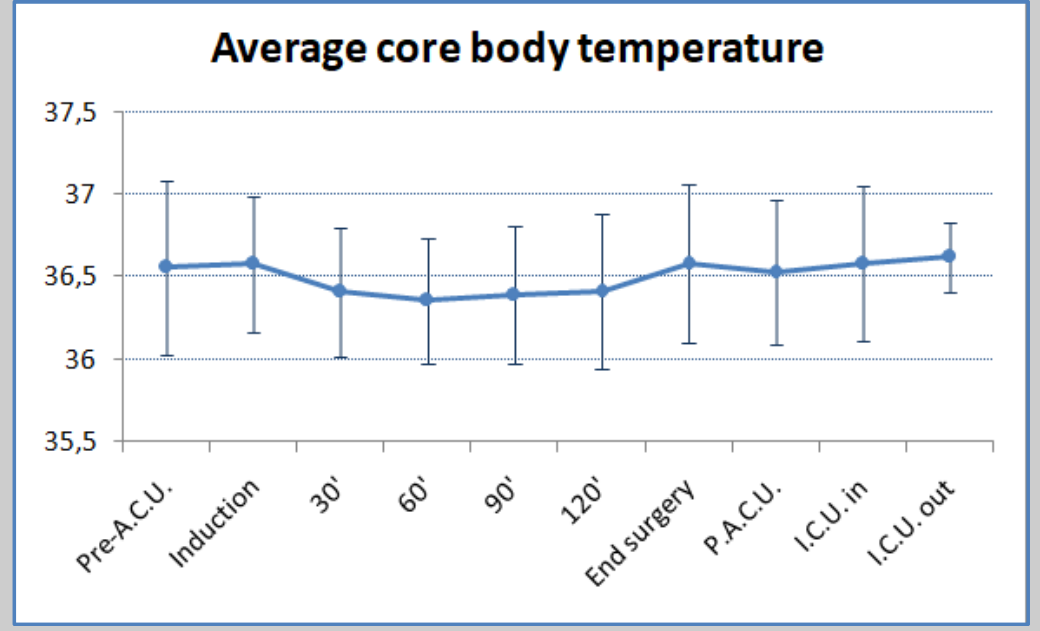

Core temperature at the end of surgery $(n=200)$
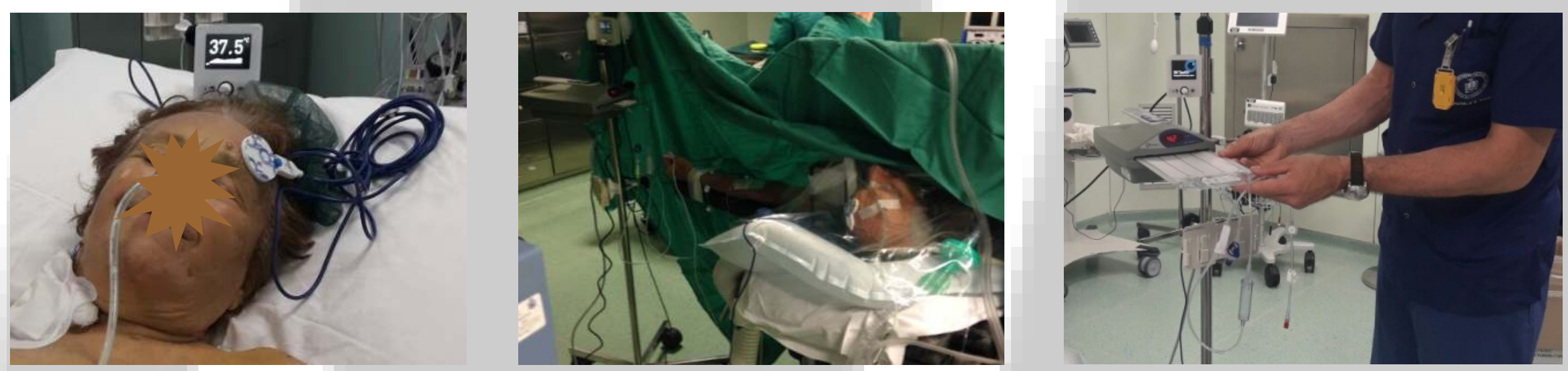

Results. In OR, at the end of the procedure, $7,5 \%$ of the patients showed a $\mathrm{T}<36^{\circ} \mathrm{C}$. Of these, 13 presented mild hypothermia, 1 moderate hypothermia, 1 deep hypothermia. Prewarming was performed on $66 \%$ of patients, the remaining $34 \%$ refused. 15 patients showed $\mathrm{T}<36^{\circ} \mathrm{C}, 66 \%$ of them were in no-prewarming group. The difference in temperature found in patients between two groups in Pre-Anestetic Care Unit and during the induction (in OR) results statistically not significant. In all the intraoperative phases and at the end of the intervention, the difference detected is statistically significant $(p<.05)$. Only weak negative correlations between the temperature $\left(<36^{\circ} \mathrm{C}\right)$ recorded at the end of the intervention and ASA, age and duration of the interventions. A case of postoperative shivering was reported, $90 \%$ of patients declared maximum comfort status and $93 \%$ felt no pain. At 5 minutes after entry into PACU, $60 \%$ of patients were completely awake and at 20 minutes, the percentage rose to $89 \%$. In PACU and in the next 24 hours, no complications were found in any patient. The average time spent in PACU was $49 \pm 21$ minutes.
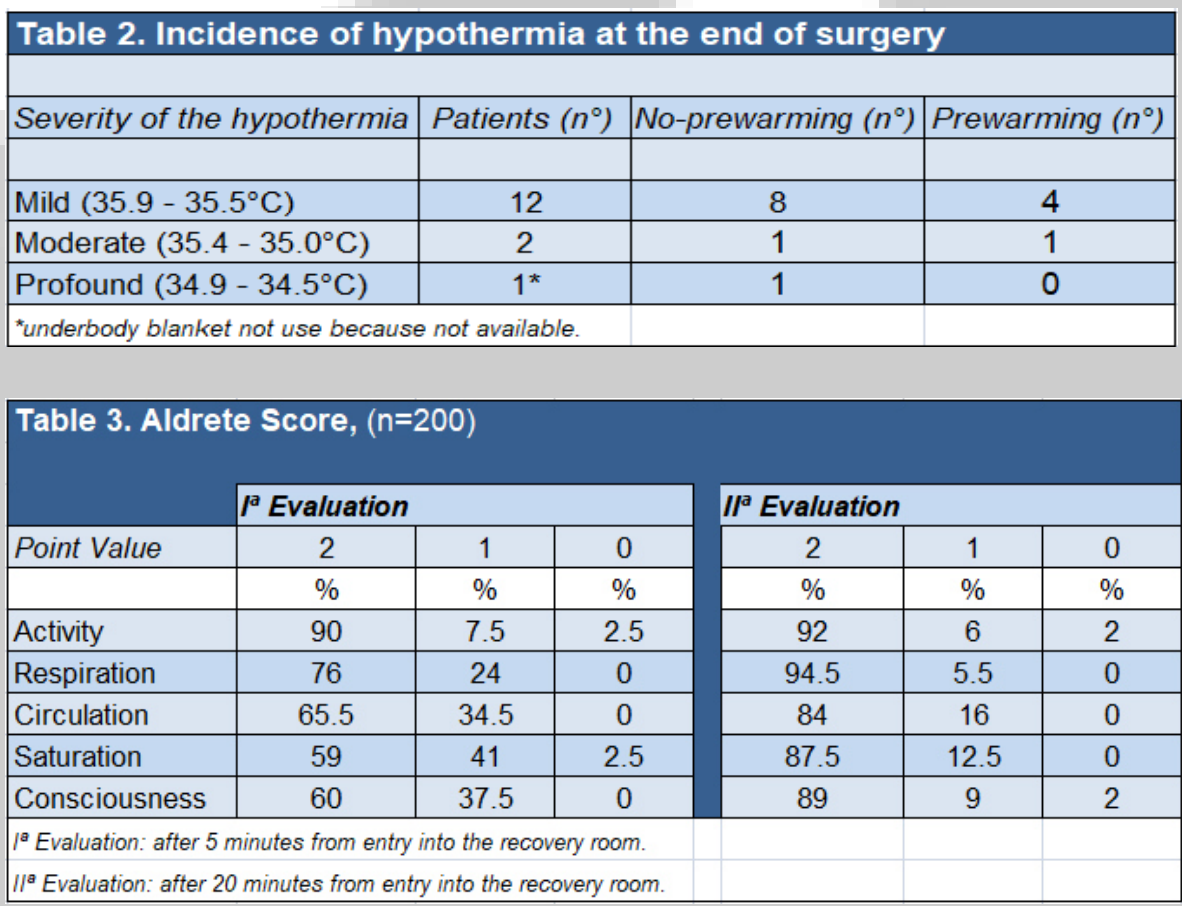

Conclusions. The analysis of the data obtained show us that the methods and treatments we have chosen for the prevention of perioperative hypothermia should be evaluated positively and in line with the expected clinical standards. Prewarming plays an important role in reducing the incidence of intraoperative heat loss and has a positive influence on patients anxiety and concern while waiting to enter OR. We have seen how the perioperative maintenance of normothermia in surgical patients determines a rapid post-operative normalization of the main vital functions as well as an equally rapid achievement of the requirements necessary for the safe discharge of the same from the operative block. The current project provides for the extension of the assistance program to all surgery specialties in our hospital with the appropriate modulations and rationalizations.In the future we also intend to evaluate the impact it may have on preventing the incidence of surgical site infections.

References

Eshraghi Y. Et al., 2014. An evaluation of a zero-heat-flux cutaneous thermometer in cardiac surgical patients. Anesth.

Horn E.-P. et al., 2012. The effect of short time periods of pre-operative warming in prevention of perioperative hypothermia. Anesth Kurtz A. et al., 1996. Perioperative normothermia to reduce the incidence of surgical-wound infection and shorten hospitalization. N Engl J Yilmaz M. et al., 2008. Impact of preoperative warming on maintenance of normothermia and outcome after colorectal surgery. Anesth. Tominaga A. et al., 2007. Efficacy on an underbody Forced-Air Warming blanket for the prevention of intraoperative hypothermia. Anesth. Tominaga A. et al., 2007. Efficacy on an underbody Forced-Air Warming blanket for the preve

Siaarti, Clinical Practice Guideline 2010. Recommendations for the recovery area and post-anesthesia care.

NICE, Clinical Practice Guideline 2008. Hypothermia: prevention and management in adults having surgery (Last update December 2016). Siaarti, Good Clinical Practices 2017. Perioperative Normothrmia.
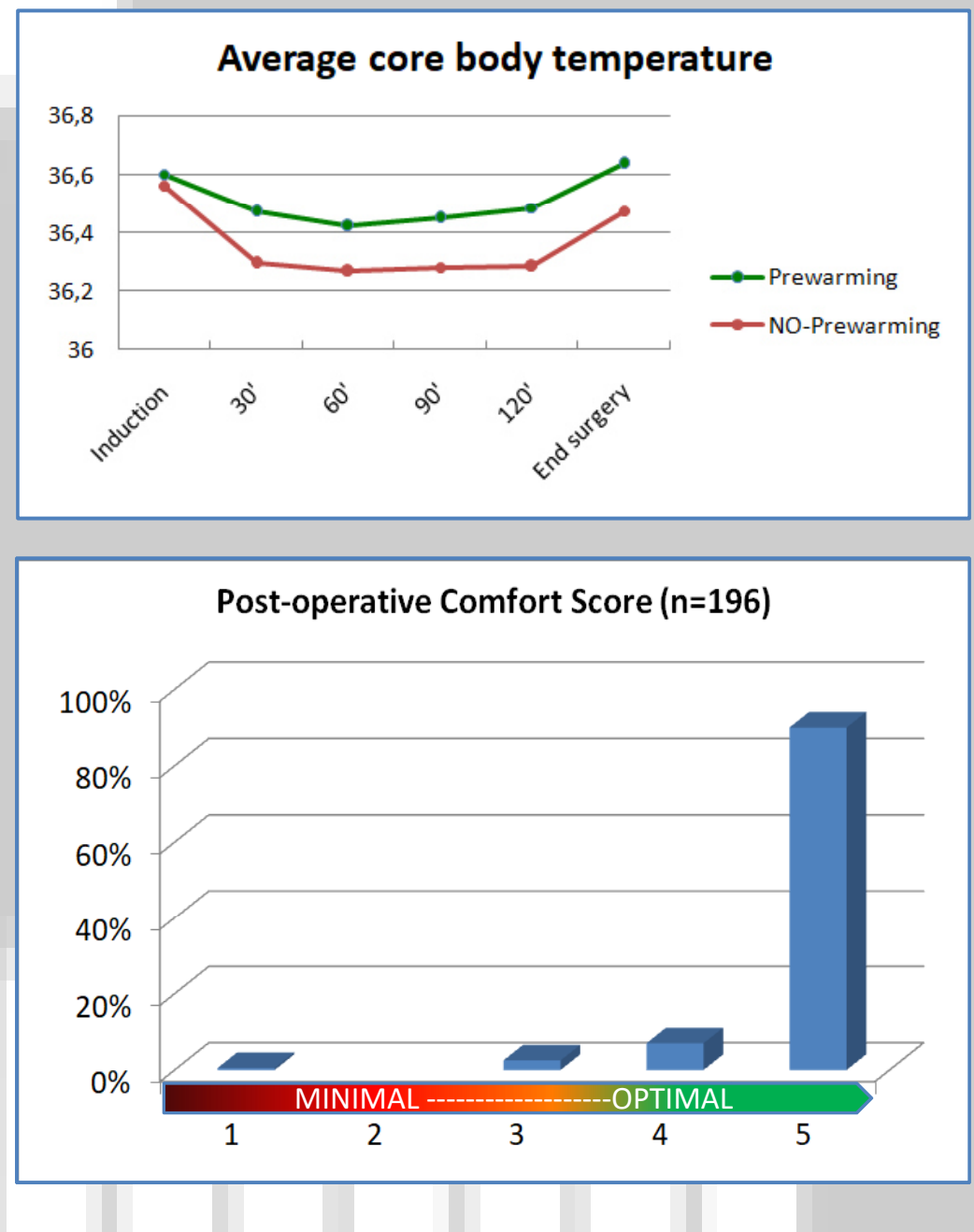

Post-operative Pain Score $(n=196)$

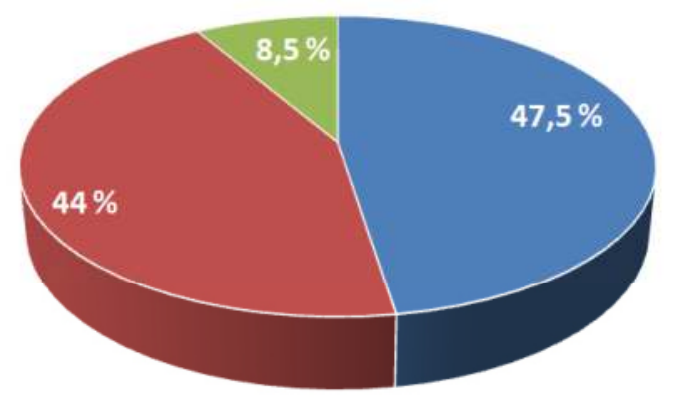

NO PAIN

- MINIMAL

MODERATE 